\title{
Out of "touch"? - An experiential pedagogical approach to daylighting in architecture and interior design education
}

\author{
Gillian Treacy ${ }^{1}$ \\ ${ }^{1}$ School of Design, Edinburgh College of Art, The University of Edinburgh, UK
}

\begin{abstract}
A new challenge is emerging. Contemporary built environment pedagogy demands engagement with both analogue and digital tools for simulation and verification of lit architectural environments. The use of analogue tools within architectural design education grasps onto the historically valued craftsmanship of drawing and physical models to measure, represent and understand our lit environment ambiance. Digital tools can provide efficient, simultaneous and precise verification of lit architectural interior space through 3D computer modelling and calculation software. However, the understanding and representation of daylit scenarios is becoming more numerically complex as lighting metrics and software gain in accuracy and dynamic range. With the majority of easily accessible software tools focussing on numerical verification, the ephemeral ambience that daylight in particular creates in interior architectural spaces is becoming ever more difficult to grasp for the architectural design student and practitioner. This paper seeks to challenge the exclusive use of digital tools for the understanding and representation of lit interiors by proposing that this methodology cultivates design epistemologies that are out of "touch". Questionnaire findings and workshop studies are presented as pedagogical constructs are proposed inviting physical, experiential learning of lighting principles in collaboration with numerical and digital modes of learning to provide connections and translations to develop through 'touch'ing light.
\end{abstract}

\section{The new challenge emerging}

Contemporary built environment pedagogy invites engagement with both analogue and digital tools for simulation and verification of lit architectural environments. An attunement to the atmosphere created by light in an architectural space is necessary for every architectural designer (architect or interior designer). This understanding of the character of light allows some certainty in the prediction of the lit ambiance of a space. However light, with its intangible and contextually dependent characteristics can be difficult to grasp and challenging for architectural designers to work with, particularly in the realm of lit ambiance. Now more than ever, a deeper understanding of the phenomenon of daylight is necessary to engage fully with contemporary holistic approaches within architectural design; light touches upon many inter-connected elements, such as building location climate, orientation, adjacent contexts, spatial volumes, materiality (such as texture, transparency and reflection), and its physiological and psychological effects on users of the space. The use of analogue tools 
within architectural design education grasps on to the historically valued craftsmanship of drawing using physical models and tools to measure, represent and understand our lit environment ambiance. However, in the field of architectural and environmental building design, engagement with digital tools is now commonplace and any architectural designer would be viewed as 'out of touch' if they avoided engagement with these technologies. Digital technologies are providing progressively more efficient and precise verification of lit architectural interior space through 3D computer modelling of virtual environments and calculation software. It is apparent that daylighting design as a generator of interior ambiance is quickly becoming a lost art in the face of numerical verification. It has become too simple to calculate light as 'energy'.

\section{As designers, we are trained to place value in the concept of spatial experience; however, we are increasingly asked to quantify our design intentions in terms of net energy balance. (1)}

This ontological understanding of light provides an alternative approach to designing with light exposed and expressed through the use of the analogue draughting tools; significant value is now given to accuracy in numbers and large data sets. Energy efficiency, lighting efficacy and solar gain can all be designed with digital tools to easily provide highly predicable data defining energy usage and compliance. It is apparent that the understanding and representation of daylit scenarios is becoming more numerically complex as lighting metrics and software gain in speed, accuracy and dynamic range. Although these data can provide some guidance on thermal comfort or even task lighting levels this provides little indication of the lit ambiance of the architectural daylit space. With the majority of easily accessible software tools for environmental analysis focussing on numerical verification, the ephemeral and embodied ambience that daylight in particular creates in interior architectural spaces is becoming ever more difficult to grasp for the design student and new architectural design professional (2). Further, quality and "uniformity of experience" (3) in interior spaces is becoming codified to ensure regulatory compliance where practical. This diminished necessity to value our personal experiences through our "implicit, naturalistic, ecological cognition of everyday existence" (4) denies powerful design motivations and limited ontologies for light.

Therefore, with energy compliance top of the agenda it might be argued that it is no longer necessary for designers to explore this phenomenological embodied relationship with the characteristics of daylight. The subtle and inviting revealing of the "thingness of light" (5) to occupants of the space is no longer a necessary attribute and skill of the architectural designer. In spite of this view, I would argue that architectural design demands an emotional understanding of spatial environments and this cannot be solely described using the daylighting metrics and tools currently available. Dean Hawkes (6), in his book "The Environmental Imagination", poses a question that invites discussion as to the place of science and technological devices in architectural design.

\section{The ability to envision the outcome of the conjunction of form and material, set within the physical facts of the climate and locale, in ways that inform and enhance the purpose and meaning of a building...lies at the very heart of the architectural project.(6)}

He proposes that the nature of architecture requires an understanding of both realms, the quantitative and qualitative, the physical and virtual, the real challenge being to understand the unquantifiable aspects of architectural design. 


\section{I only wish that the first really worthwhile discovery of science would be that it is recognised that the unmeasurable is what they're really fighting to understand. (7)}

Pallasmaa argues that "The quality of a space or place is not merely a visual perceptual quality as it is usually assumed. The judgement of environmental character is a complex multisensory fusion of countless factors, which are immediately and synthetically grasped as an overall atmosphere, feeling, mood or ambiance" (3). Plummer (8) advocates through photographic and written narrative research that daylight heightens the "immaterial aspects of architecture" and the human experience of being in a space touches "perceptual", psychological", "emotional" and "spiritual" realms. He suggests that this emotional experience of light in a space is a necessary personal exploration for anyone aspiring to gain insights into daylighting. With this in mind, it can therefore be suggested that the architectural designer needs an ever expanding and flexible skillset benefitting from the ontologies of both the quantitative 'science' and qualitative 'art' of light. The increasing variety of tools, outputs and competencies and their understanding in use demands an "eclectic and heterogeneous skills set" (9) with an underlying knowledge of the characteristics of daylight to use the tools available effectively.

This paper therefore suggests that the educational methods to invite authentic understanding of the particular phenomenon of daylight are critical, valuable and necessary. It is proposed that an understanding of and the ability to predict lit ambiance cannot be disregarded in architectural communities and educational establishments in favour of tools that allow for numerical building design energy output data sets or 'optimised' solutions with little engagement or skill in their creation and complexities in their interpretation. The design tools used for the analysis of daylighting in architectural contexts currently generate unrecognisable data for architectural designers (see questionnaire results below), as outputs give little indication of the architectural space and the contributing factors directly affecting the results. The understanding of this data requires translations into 'designerly ways of knowing' (10) to ensure intentions are understood and the ability of architectural designers in the understanding and prediction of lighting ambiance does not become a lost skill or undervalued architectural design element.

\section{As long as educational frameworks relegate the emotional and experiential to the place of a supplement, then our design processes will continue to unconsciously promote environments of thinness and superficiality. (11)}

It is therefore proposed that tools, providing primarily quantifiable data are in themselves 'out of touch' with design methodologies. They do little to assist designers with the prediction of important insights into lit environment ambiances that relate to increasing understanding in the field of physiology and psychology of the visual and non-visual effects of light. This paper seeks to challenge the exclusive use of either method, whether analogue or digital, for the understanding and representation of lit interiors. Either methodology used in isolation encourages the cultivation of design epistemologies and ontologies for light that are out of "touch". Instead, this paper aims to rise to the challenge of these contemporary complex understandings of daylight, the science and the art of the light and questions: If the demand for metrics to predict the energy value of daylight continues to grow how might this ontology of daylight and the lighting data generated help architectural designers to improve their understanding of lit ambience of a space? Findings from a questionnaire are presented to support the case for understanding and interpretation of quantifiable measures of daylighting in architectural communities. Further, pedagogical constructs based on the problems 
identified in the questionnaire are evaluated through findings from a series of educational workshops that invite physical, experiential learning of daylight's characteristics in collaboration with numerical data and digital modes of learning to provide connections and translations (12) to develop.

The very character and purpose of light is dependent on a set of design principles which are revealed to the observer through experience, and not through a planar map of illumination levels. (1)

\section{Translational Explorations - Design Practitioner's Questionnaire}

This paper first reports on the findings from a questionnaire of over one hundred architectural professionals in the UK. The questionnaire was initially created to explore the broader conversation of how architectural designers perceive architectural lighting information and to determine a common knowledge base or ontology for light across varying scales of architectural practice and communities. The questions were created by introducing a series of visuals to view on an individual's computer screen. Each visual example provided a different format and level of lighting information to ensure a fairly broad range of contemporary lighting representations/information outputs were tested. Responses to these visuals were recorded by each individual designer as a series of qualitative descriptive written answers to branched, open-ended questions. It was hoped that through analysing these descriptions of the visuals some understanding of the common or diverse interpretations of architectural lighting from analogue and digital representations of lit spaces would emerge.

The questions were created as paired visuals; representations of the lighting of interior spaces. Each question displayed a simple visual showing one representation of lighting information. The following question of the pair added an additional layer of lighting information. The participants were asked to respond to each question in sequence and not to return to a previous question. This guidance was to ensure parity in the sequencing of responses and to guarantee responses were not affected by information gained in later or the second of the pair of questions. This paper discusses the findings from the first four example questions presented.

The selected examples utilise plan views of iconic architectural spaces, one of those selected is discussed in this paper; le Corbusier's Notre Dame du Haut, in Ronchamp, completed in 1955. This building was selected for its notoriety and familiarity in architectural communities in Western Europe (the participant group were UK based) and, most importantly, its large variation in daylighting conditions and ambiance. It was expected that any architectural designers familiar with the work of Le Corbusier would be able to identify the building by its unique plan form and elevational profile. The visual shown in the questionnaire therefore takes the form of an isolux diagram as an orthographic plan projection where contours provide points of equal illuminance. This format of a plan with contour lines is representational of the varying daylight levels throughout the space as calculated on the working plane ${ }^{1}$. Rather than Illumination levels (Lux) being shown, the plan indicated Daylight Factor (DF) ${ }^{2}$ numerical values, expressed as a percentage, a visual format used by many daylighting and environmental design software applications. The Daylight Factor is a metric used in the UK and defined by UK building regulations to predict expected daylight

\footnotetext{
${ }^{1}$ The 'working plane' as defined in https://www.cibse.org

${ }^{2}$ Daylight factor as defined within https://www.cibse.org
} 
levels. Although this metric is now under scrutiny for its accuracy in use ${ }^{3}$ it was assumed that DF values often demanded as part of local authority planning and building control guidance would be a familiar lighting metric to many architectural designers in the UK. Of the 110 respondents to the question, only $10 \%$ were able to correctly describe the metric used as 'Daylight Factor' and/or most commonly, incorrectly used the term 'luminance' in their response. When asked to discuss the predicted lighting contrast in the space only two of the respondents identified with the numerical characteristics of the isolux diagram and were able to note ratios of 1:5 etc.

The second example used the same plan showing the same Daylight factor values but added a graded white to black monochromatic shading render between each contour relating to the DF values: high (white) to low (black). In analysing the responses, it quickly became apparent that with this additional layer of information it was possible for all those examining the visual to arrive at similar conclusions for the descriptive writing task. Significantly, using the combined rendered and numerical visual, $90 \%$ of the participants felt confident enough in their understanding to answer this question and $60 \%$ of participants discussed the contrast using the numerical information given on the plan. These data highlight clearly that the visual format, including the shaded patterning helped significantly in the translation of the numerical DF information.

The second pair of questions was selected to show a virtual view of the same built space (Notre dame du Haut) created using Sketch-up. For continuity, the same building was chosen and the same main space visualised but in this scenario the format was a virtual representation of the space with shadows turned on and materials as defined by the limitations of the software's materials palette library. The same questions were asked and the results were considerably different. It was assumed that participants would at this point realise that the space they were considering was the same one as that in the previous questions, but it is clear this was not the case. Participants described the space in terms of volume and only $5 \%$ of respondents wrote more than a couple of words describing the lighting. The second visual of the pair had luminance information added as measured on site at the time of the photo being taken. Luminance levels were measured with the use of Photolux app ${ }^{4}$. Many more participants responded to this visual and notably $30 \%$ discussed the lighting contrast. It was particularly interesting to note that the Sketch-up visual did not show as significant contrast values (to see by the eye) as the numerical values displayed and yet all the descriptions suggested that there was 'little contrast', or the space was 'evenly lit', even although the measured contrast was 1: 5 and were noted as such on the visual. It was apparent that although this numerical information had been added to the visual the actual numbers shown either weren't understood or the participants had an overriding bias to the visual scene shown rather than translation of the numerical results.

The final pair of questions aimed to define a descriptor of the lit ambiance of the space using a photographic visual of the interior. Again, for continuity, the same building was chosen and the same main space visualised but in this scenario the format was a simple colour photograph of the space. All participants responded to this visual and responses used many verbs and adverbs to convey the lighting. When asked to describe the contrast in the space all attempted to discuss the contrast in relation to the architectural characteristics such as; "sharp light through the small piercing windows against the gloomy wall', 'tiny windows provide strong contrast', 'the dark areas are contrasted with the very bright clerestory'. The context of the lighting and its relationship to the built space was clearly helpful in providing

\footnotetext{
${ }^{3}$ Sketch-up - https://www.sketchup.com

${ }^{4}$ https://www.photolux-luminance.com
} 
an ambiance that could be visualised and described easily by the participant group. The second image of the pair used the same base photograph but added in luminance levels as measured on site at the time of the photo being taken. Luminance levels were measured with the use of Photolux app. With this added information $70 \%$ of participants chose to discuss the numerical contrast values alongside the text descriptors. Again, for this visual image, the added layer of luminance values provided further information to discuss but was clearly understood as a metric in relation to the view of the interior space.

These findings, although limited due to the participant groups locality (UK based) and number of participants, highlighted points worth investigating further. Respondents showed little awareness of lighting metrics, their numerical relationships and the technical terminology associated with daylighting. It must be presumed that either these architectural designers do not have a need for this knowledge or they are passing these aspects of the design on to another. Were the numeric values simply not understood? Research has shown that our understanding of lit ambiance cannot easily be equated to photometric values (13). As daylighting metrics are derived from schema that are fundamentally mathematical constructions, not physical luminous quantities, (14) it can be challenging to predict the temporal, context specific phenomenon of daylighting ambiance in an interior space.

The results may imply that the rationale behind lighting metrics is not clear and, significantly all the more confusing as we lose our direct engagement with daylight's intrinsic characteristics concealed behind a digital user interface. Digital methods allow data to be generated seamlessly and few architectural designers have shown this to be problematic but clearly the translation of this information into useful project information is less successful.

Many software developers are researching solutions to create improved speeds and accuracy to encourage designers to engage better. However, few architectural designers have reported that the speed of calculation is problematic. Rather, it seems that this research and development may be misaligned. The results of the questionnaire suggest that the output data from the software would be more frequently used if it could be understood and therefore assigned more value by designers as an important part of their design process. The hidden processes that generate the lighting data in digital software may be better understood if exposed to the user in an intermediate form, rather than concealed behind the user interface. As soon as the metric formula with its relatively straightforward relationships, angles of the sun to sill height and areas of window to wall are removed from sight we seem to lose our understanding of how the numerical outputs are generated and the rationale behind the metric itself. In order to understand daylighting software output data fully it is crucial that the designer is given the full story, for example; what parameters were used for this calculation and what values were assumed? This is important information, for without the designer understanding these input values it is impossible to analyse results and revise the design appropriately to suit the architectural rationale and design priorities. 


\section{A need for experiential explorations}

In Pallasmaa's essay on 'Multisensory experience: The significance of Touch', he suggests that genuine architectural works stimulate our ideated sensations of touch, and this stimulation is in itself life-enhancing as it enhances our experience of ourselves (3). This idea of a physical encounter through ideated sensations when we interact with an architectural space has support in contemporary scientific findings. Discoveries reveal a more embodied understanding of emotion, through our sensorimotor circuits providing mirror mechanisms resulting in "embodied simulation" (Gallese 2005 in (15), the physiological rationale behind our enjoyment of watching dance, examining art or listening to music being played. This connection with actions and the environment within which they are taking place can be aligned with our appreciation of an architectural space, its character and the emotions it evokes as we experience it. It suggests too that as we perceive daylight patterning in an architectural space; the ephemeral, transient nature of daylight interacting with the architectural materiality and spatial composition we attune sensorialy and emotionally with these fluctuating surroundings as Edmund Husserl describes "animate organisms". This paper proposes that design educators can learn from Pallasmaa's call to defy this "sensory impoverishment" and Mallgrave's Phenomenological Model for Architectural Research by re-evaluating daylighting pedagogy through valuing experiential learning with regard to lit ambiance.

The following outlines an investigation into a contemporary pedagogical approach to daylighting design that seeks to make transparent the ephemeral and particular ambiance that daylight can create in architectural interior spaces through strategies that encourage "sensory invitation and discovery, mystery and shadow" (16). Using a Constructivist pedagogical approach phenomenological constructs are proposed that align with teaching methods to reengage with daylighting in experiential ways, allowing designers to "work directly with the object of their thought" (12). Designed workshops aimed to encourage sensory invitations to discover daylighting by re-engaging physically with context specific scenarios. Strelitz (17), anthropologist and interior designer, when discussing her approach to creating 'Buildings that feel good' notes that 'Learning from buildings that feel good instructs us in creating buildings of lasting value... as elements with which we engage in the built fabric around us"((17) p3). As we are often unaware of lighting in a room unless it is particularly poor, it was considered important to discuss the rationale of each workshop with participants beforehand to ensure that the processes they became involved with were explicitly and consciously considered. Theodorson (18) has highlighted the difficulties in teaching lighting in this regard:

\section{Humans have untold experiences with light; it is the primary means we use to "know" our world. Yet this immense range of exposure is primarily passive and ironically, does not often result in retrievable knowledge about light's physical behaviors, impacts, qualities, or intensities. (18)}

The workshops outlined in this paper challenge participants to "touch" light, implying a physical and emotional connection with daylighting that the study questionnaire suggests we intrinsically value. They sought to provide practical strategies for the unravelling of daylighting design through overlapping digital and analogue methods and how this might enhance understanding of the lit ambiance of a space in current contexts. 


\section{Translational explorations - 'touch'ing light workshop}

A series of workshops was created to explore and evaluate physical, haptic engagement with light. The research explorations utilised design workshops, focus group discussions and questionnaires with the aim of identifying insights into design processes and knowledge acquisition through the application of varying tools in daylighting design scenarios and the subsequent participants' responses. Design studio was chosen to test these methods through controlled scenarios and observational techniques. Studio workshops were designed to fit within a current architectural educational setting to ensure continuity of results through weekly analysis within the same environmental setting and participant group, producing results from real studio contexts without the added complexity of unfamiliar, simulated situations.

Over a period of a month, a group of approximately 30 students attended a two-hour workshop in lighting each week. The student group was a mix of $1^{\text {st }}$ year Interior design students to Masters level Interior design students ranging in nationality and design educational background. Participants were placed into groups of three to encourage discussion and provoke realisation of others values and experiences of the lit environment. The workshops were held in the general studio space, providing access to laptops, model making materials, drawing boards and layout space. Attendance was optional but encouraged. The work created was not assessed but it was assumed that any drawing, model or design work generated would be beneficial for each student's portfolio. The first of these series of workshops is outlined here:

Workshop 1 - Describing lit environments -photographic visuals of an architectural space Describing lit environments -photometric visuals of an architectural space

Workshop 2 - Creating matching lit environments (2D visual to 2D drawing) through hand drawing

Creating matching lit environments (2D visual to $3 \mathrm{D}$ drawing) through physical modelling

Workshop 3 - Measurement of Illuminance and DF using software and 3D VE model Adapting 3D VE models to comply with metric requirements

Workshop 4 - Measurement of Illuminance and DF using physical modelling Adapting physical models to comply with metric requirements

Workshop 5 - Focus group meetings

The workshops provided an opportunity for students to work in an experimental way using a broad range of approaches to describe a lit environment. The pedagogical approach of weekly group tasks commenced with shared oral and written descriptions of daylit interior environments followed by a re-creation of one of the spaces as imagined in a threedimensional physical model to allow open discussions around the subject of daylight, to awaken understanding of others' unique understanding of lit ambiance and common qualitative aspects of daylighting design.

A transformation in the architectural design process should be stimulated redirecting attention to the context and the cultural spirit of 


\section{place, as well as encouraging a sound understanding of the quality of natural light. (19)}

Through three-dimensional heuristic learning methods and associated formats for expression it was imagined that participants would indeed learn to 'touch' light tempted by the invitation to explore and investigate lit space. The subsequent workshops required engagement with daylighting metrics, using quantitative methods of analysis. Participants were provided with a three-dimensional virtual model of their space to work with in IES VE software and further to a 15-minute demonstration tutorial, each small group followed through calculating the daylit environment using basic sunlight and daylight metrics. This quantitative analysis was then repeated using each group's physical model measuring DF, illuminance and luminance with Edenapp and Photolux apps on mobile phones placed within or against the physical model. These workshops demanded physical model making to encourage haptic engagement with lighting metrics.

The final workshops asked for revisions to both proposals, the three-dimensional virtual model and the three-dimensional physical model. Participants were asked to revise the models physically and virtually to comply with a $4 \%$ daylight factor and an average illuminance of 200 Lux over the working plane as an example level requested by local building regulations.

Interior lit environments were selected to be broad ranging yet architectural projects that had a strong interior and daylit aesthetic. Student participants were able to describe the photographic visuals well. Very few were able to discuss the photometric visuals successfully and no participants commented that the same space had been shown in qualitative and quantitative terms. It was interesting to note that the students used a similar amount and type of descriptive language as the practitioners who had been given the same lit interior scenario in their questionnaire.

The participants created physical models of an interior space based on the 2D visual they had been given. Participants worked quickly and confidently to develop spatial voids which they filled with texture, colour and light. Some participants used transient sunlight to explore ephemeral atmospheres. Others used static artificial light and layered the spatial armature with tones and textures to correspond with their understanding of the space. Most models created had no determined scale but were successful nonetheless in realisation as it quickly became apparent to the participants that light has no scale. Many models had a clear observation angle. Conversation analysis during group work made this phenomenon explicit as participants declared "You have to stand here to see it properly!" Most physical models allowed for participation within the spatial void created. Other participants were invited to "come, look inside and see how it feels!"

Participants found it quick and straightforward to create photometric data for their 3D virtual environment model. The output data as Isolux diagrams, numeric DF values and contrast ratios could only be explained by a few participants however. The lack of understanding of these numbers and the relational values to the interior space and external context was further demonstrated in the subsequent workshop.

5 IES ve - https://www.iesve.com/ 
The following week, participants were asked to adjust their VE model to suit the metrics given for compliance. Participants were unclear as to which input variable to select but very quickly this approach, allowing multiple trial and error scenarios to be evaluated, showed some success in the numerical results. Significantly it was noted by most that the reflectance of ceiling material became crucial to the metric values achieved. When participants were asked to discuss how this change in reflectance might be created in a real project with real materials most assumed the use of mirrored surfaces rather than changing the colour (light absorption properties of the material). It became clear that the participants were making changes to their design without really reflecting or understanding the nature of their revisions and the impact on the architectural aesthetic or change to spatial perception in achieving the required results. Nicholas Carr, in writing an article about on-line learning, discusses controversially that in using digital software we are frequently just 'decoders'(20), considering unrelated parts of information out with known contexts. The findings of this study imply that this may be happening when we use digital software without a basic knowledge of the processes involved. It is proposed that perhaps the lack of haptic engagement presents itself in this specific situation through observing this virtual scenario created using digital lighting software devoid of experiential context. Pallasmaa (11) proposes that "touch is the unconsciousness of vision, and this hidden tactile experience determines the sensuous qualities of the perceived object".

In the second part of the workshop, students sought to measure the lit ambiance of the physical model photometrically (Fig.1.). Students used Edenapp and Photolux to calculate illuminance levels, luminance and DF values. Participants were then asked to adjust these measurements to suit a given lighting metric requirement through manipulation of the model. It became apparent very quickly that this was possible and connections were strong linking the metrics with the physical model materiality, opening sizes etc. Each causal architectural relationship within the architectural space needed consideration and this was easily tested using physical materiality and light source.

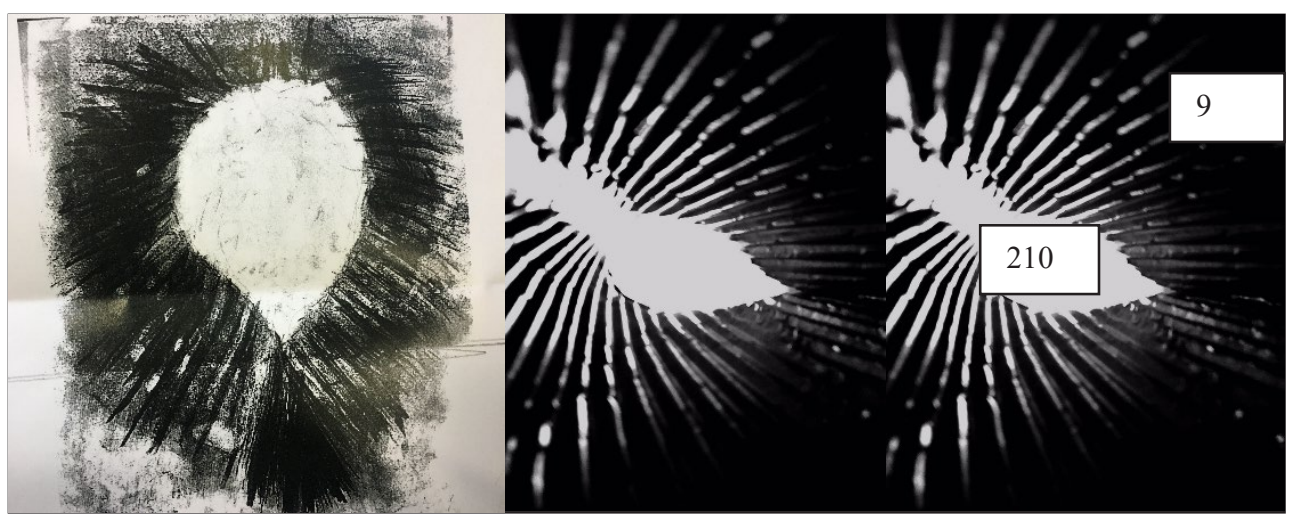

Fig. 1. Example drawing to model image to Photolux layer. Source for exploration Bruder Klaus Chapel - Zumthor

Each workshop was discussed in the subsequent focus group sessions and a key element of daylighting knowledge and design epistemology emerged. The exploration of these relationships between light and architectural space served to help visualise this embodiment explicitly. It was necessary for these design explorations to be interactive and encourage iterative variables in order to unravel the varying relationships between materials, light and photometric values. 


\section{Future explorations}

In conclusion, this study offers insights into methodologies for design pedagogy that can provide transparent relationships between experiential learning, numeric reasoning and representational techniques seeking to encourage the creation and translation of daylit ambiances through overlapping of each mode with numerical digital interactions. Through the demonstration of multi-modal tools in use, this paper proposes future collaborations to reveal understanding of ambiance through experiential design. This application of qualitative and quantitative measures and insights may provide improved understanding of daylight's characteristics and potential in built environment contexts. In so doing this provides a better understanding of lighting metrics and their use in application. This methodology of practice is wide ranging and may provide a basis for further research addressing collaborative pedagogies within related fields of architecture with acoustic, sound design and other intangible sensory applications.

\section{Becoming more fully aware of the extent of our biological complication, whose underpinnings reach deeply into the sensory- emotive world that we daily inhabit, is simply a first step in this process". (21)}

This research seeks to challenge the idea that we may be out of 'touch' experientially when we use digital tools exclusively rather than, as might be more commonly understood, as being out of 'touch' by not using digital methods. The current tools available for daylighting design can meet the expectations of contemporary design students and educators if used in parallel and in haptic and collaborative ways. Using a combination of tools, the architectural designer might predict the lit ambiance of an interior space but only through first understanding how the relationships of light and its interactions with the interior space work - characteristics as experienced. Only with this understanding can a sensitivity to daylighting ambiance be revealed and intentionally designed into our built environment.

The computer is an important tool-no one could do without it - but for me it's only a tool and it doesn't replace thinking. It can make you disconnected...bring it out of the computer...use physicalities and models to understand and anticipate what this thing will be in the end: something physical, something real, something for people. (22)

\section{References}

1. Rockcastle S. Annual Dynamics of Daylight Variability and Contrast A SimulationBased Approach to Quantifying Visual Effects in Architecture. Andersen M, editor: London : Springer London : Imprint: Springer; (2013)

2. Treacy G, Innovative learning technologies for daylighting education. Professional Lighting Design Convention; Rome: VIA Verlag (2015)

3. Pallasmaa J. Encounters : architectural essays. MacKeith PB, editor. Helsinki: Helsinki : Rakennustieto Oy, (200)

4. Storkerson P. Naturalistic Cognition: A Research Paradigm for Human-Centered Design. Journal of Research Practice. 6(2):M12.(2010)

5. Holl S. Parallax. Basel: Basel : Birkhäuser; 2000. 
6. Hawkes D. Environmental imagination. London: London : Routledge; (2008)

7. Lobell J. Between silence and light : spirit in the architecture of Louis I. Kahn. Kahn LI, editor: Boston ; London : Shambhala; (2008)

8. Plummer H. Poetics of Light. A $+\mathrm{U}$ (Architecture + Urbanism) Extra Edition.(December no.12). (1987)

9. Mardaljevic J. Book review: Daylighting handbook I. (2016)

10. Cross N. Design thinking : understanding how designers think and work. Oxford: Berg; (2011)

11. Teal R. Dismantling the Built Drawing: Working with Mood in Architectural Design. International Journal of Art \& Design Education. 29(1):8-16 (2010)

12. Evans R. Translations from drawing to building. AA files. (1986)

13. Boyce PR, Smet KAG. LRT symposium 'Better metrics for better lighting' - a summary. Lighting Research \& Technology;46(6):619-36. (2014)

14. Tregenza P, Mardaljevic J. Daylighting buildings: Standards and the needs of the designer. Lighting Research \& Technology.50(1):63-7 (2018).

15. Harry Francis M. Embodiment and Enculturation: The Future of Architectural Design. Frontiers in Psychology. (2015)

16. Pallasmaa J. Hapticity and time: Notes on fragile architecture. The Architectural Review. 207(1239):78-84.( 2000)

17. Strelitz Z. Buildings that feel good. London: London : RIBA; (2008)

18. Theodorson J. Teaching light - Constructing knowledge across multiple dimensions. (2012)

19. Traverso G, editor. Modelling Daylight. Germany: VIA Verlag; (201).

20. Carr N, Norvig P. Is Google Making Us Stupid? New York Times Upfront.143(3):22. (2010)

21. Mallgrave HF. The architect's brain : neuroscience, creativity, and architecture. Wiley I, editor. Chichester, West Sussex, U.K. ; Malden, MA; Wiley-Blackwell; (2010)

22. Gordon A. "Credit Swiss," Interview with Pierre de Meuron and Jacques Herzog. The Magazine from the Wall Street Journal. (2008) 